\title{
Selective Reduction of C-C Double Bonds of 2-Vinylaziridnes: Preparation of Enationmerically Pure 2-Alkylaziridines
}

\author{
Baeck Kyoung Lee, Bong June Sung, Won Koo Lee, Doo-Ha Yoon, ${ }^{\dagger}$ and Hyun-Joon Ha ${ }^{\dagger *}$ \\ Deparment of Chemistry. Sogang Lniversity, Seoul 121-7+2. Korea. E-mail: wonkootasogang ack \\ ${ }^{\dagger}$ Deparment of Chemistry and Protein Research Centre for Bio-Industry, Hankuk Lniversitv of Foreign Studies, Iongin, \\ Kunggi-Do 4+9-719, Korea. "E-mail: hihaiâhufs.ack \\ Recened Atrgust 6, 2009, Accepted October 23, 2009
}

Key Words: Chiral aziridine. Selective reduction. Heterocycles, $\beta$-Amino alcohol, Unnatural amino acid

The synthetic utility of aziridine, nitrogen-containing thee membered ring. stems from the regio- and stereoselective ringopening reactions with the proper side chains attached to the ring. ' Since we have developed the preparation of enantiomerically pure $\mathrm{N}-\alpha$-methy lbenzy l-(2R)- and (2S)-aziridine-2-carboxylates, ${ }^{2}$ it is possible to achieve the asymmetric synthesis of diverse nitrogen-containing compounds including amino acids, amino alcohols, diamines. and many heterocycles via functional group transformation of carboxylates and aziridine ring openings (Scheme 1).

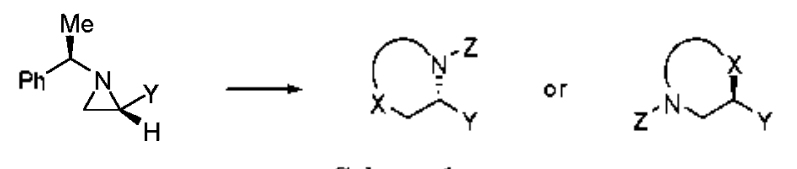

To enrich the utility of enantiomerically pure (2R)- and (2S)-aziridine-2-carboxylates, it is necessary to develop a proper method for the preparation of 2-alky laziridine which has various functional group in the side chain. The substitution of 2toluenesulfony loxy methyaziridne with alkyl nucleophiles has been studied to result limited success. ${ }^{4}$ Only one isomer I-[1'(R)$\alpha$-metlyylbenzyl]-(2S)-(sulfonyloxymethyl) aziridines reacted with dialkylcuprates to give the expected alky lation products while the other isomer (2R)-(sulfonyloxymethyl) aziridine did not provide the coupling product. The conformational restriction of the bulky $\alpha$-methylbenzyl group on the ring nitrogen seems to limit the accessibility of nucleophiles toward the reaction site. Another option to obtain 2-alkylaziridine is the selective reduction of vinylaziridines ${ }^{5}$ which can be prepared from the olefination of $1-\alpha$-methylbenzyl-(2R)- and (2S)-aziridine-2carboxyaldehyde. However there are three potential reduction sites in the substrates including $\alpha$-methylbenzyl group. allylic activated aziridine ring $\mathrm{C}(2)-\mathrm{N}$ bond. and double bond in the side chain. Due to the high strain energy of the aziridine ring. it can be reduced by many reductive conditions. ${ }^{\circ}$ Additional allylic activation by the double bond makes the ring much more vulnerable to reduction conditions. Since there has been no report for the selective reduction of $\mathrm{C}-\mathrm{C}$ double bonds of 2-vinylaziridines for the preparation of enatiomerically pure 2-alkylaziridines. we would like to describe the selective reduction method with its synthetic scopes.

\begin{tabular}{|c|c|c|c|c|}
\hline $\mathrm{Ph}^{\prime}$ & $L_{N R}^{N}$ & $\begin{array}{r}\mathrm{NBSH} \\
\mathrm{Et}_{3} \mathrm{~N}(8 \\
\mathrm{CH} \\
0^{\circ} \mathrm{C}-\mathrm{r}\end{array}$ & iiv.) & $\mathbf{R}$ \\
\hline Entry & Configuration & of $\mathrm{C}-2$ & $\mathrm{R}$ & Yield $(\%)$ \\
\hline l & $S(1 \mathbf{a})$ & & $n$-Pr $(\mathbf{2 a})$ & 83 \\
\hline 2 & $S(1 b)$ & & $n$-Hex $(\mathbf{2 b})$ & 99 \\
\hline 3 & $S(1 c)$ & & Dodecane $(2 c)$ & 78 \\
\hline 4 & $S(1 d)$ & & $\mathrm{Ph}(\mathbf{2 d})$ & 91 \\
\hline 5 & $S(1 \mathbf{e})$ & & $p$-Tolyl (2e) & 64 \\
\hline 6 & $S(1 f)$ & & $4-\mathrm{Cl}-\mathrm{Ph}(2 \mathrm{f})$ & 72 \\
\hline 7 & $S(\lg )$ & & $4-\mathrm{NO}_{2}-\mathrm{Ph}(2 \mathrm{~g})$ & 71 \\
\hline 8 & $S(1 \mathbf{h})$ & & $2-\mathrm{I}-\mathrm{Ph}(2 \mathrm{~h})$ & 95 \\
\hline 9 & $S(1 \mathbf{i})$ & & $2-\mathrm{F}-4-\mathrm{Br}-\mathrm{Ph}(\mathbf{2} \mathbf{i})$ & 70 \\
\hline 10 & $S(1 j)$ & & $\mathrm{CN}(2 \mathbf{j})$ & 78 \\
\hline 11 & $S(1 \mathbf{k})$ & & COOEt $(2 \mathbf{k})$ & 78 \\
\hline 12 & $\mathrm{R}\left(\mathbf{1}^{\prime} \mathbf{a}\right)$ & & n-Pr (2’a) & 82 \\
\hline 13 & $\mathrm{R}\left(\mathbf{1}^{\prime} \mathbf{b}\right)$ & & $\mathrm{Ph}$ (2'b) & 83 \\
\hline
\end{tabular}

Scheme 2

At first hydrogenation methods were applied with various transition metal catalysts including $\mathrm{Pd} . \mathrm{Pt}$. and $\mathrm{Rh}$ for the selective reduction of the olefin in the 2-vinylaziridine substrate to y ield the aziridine $\mathrm{C}(2)-\mathrm{N}$ bond reduced products due to the formation of $\pi$-allyl complex in all cases. ${ }^{8}$ Then we looked into reduction conditions without using transition metals and found that dimide would be a suitable reagent for the selective reduction of the double bond without reducing ring $\mathrm{C}(2)-\mathrm{N}$ bond. Since the aziridine is very labile in acidic condition to give the corresponding ring opening product we used decomposition of ary lsulfonylhydrazine in the presence of a base to generate diinude. ${ }^{9}$ Using arylsulfonylhydrazine provided a promising result to yield the expected double bond reduction product in less than $50 \%$ yield with some decomposition products due to harsh reaction condition. However. $o$-nitrobenzenesulfonyl-hydrazide (NBSH) was known for a convenient precursor of diinide since the $o$-nitrobenzene-sulfinate is a good leaving group under mild condition. ${ }^{1 / 1}$ The reaction of 2-vinyl aziridines with NBHS in the presence of triethylamine in $\mathrm{CH}_{2}$ $\mathrm{Cl}_{2}$ provided only $\mathrm{C}-\mathrm{C}$ double bond reduction products without 
influencing other functional group in the side chain. The reaction was quite successful with all $1-\alpha-m e t h y l b e n z y-(2 S)-(2-$ alkylvinyllaziridines including $n$-hexyl. and tridecanỵl in 99. and $78 \%$ yields. 2 -Aryl substituted vinylaziridines with phenyl. p-tolyl. 4-chlorophenyl. 4-nitrophenyl. 2-iodophenyl and 4biphenyl at the aryl position yielded the expected double bond reduction products in more than $70 \%$ up to $95 \%$ yield. This protocol was applicable to the isomeric substrates $1-\alpha$-methylbenzyl-(2R)-(2-alkylvinyl)aziridines with $n$-propyl and phenyl substituent in 82 and $83 \%$ yield respectively. This method was highlightened by the selective double bond reduction of aziridine-2-acry late in quantitative y ield. The similar aziridine-2acrylonitrile was reduced to 2 -cyanoetlyylaziridine in $78 \%$ yield. The selective reduction product (2k) was hy'drogenated in the presence of $\mathrm{Pd}(\mathrm{OH})_{2}$ to yield $5(\mathrm{R})$-methylpyrrolidin2 -one (3) ${ }^{\mathrm{ll}}$ from the selective reduction of $\mathrm{C}(3)-\mathrm{N}$ bond of the aziridine ring. debenzylation and subsequent intramolecular cyclization in $91 \%$ yield.

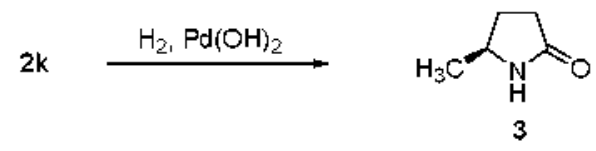

Scheme 3

In this note an efficient $\mathrm{C}-\mathrm{C}$ double bond reduction method of enantiomerically pure 2-vinyl substituted aziridines was described with NBHS in the presence of trietlylamine in $\mathrm{CH}_{2} \mathrm{Cl}_{2}$ without breakage of aziridine ring bearing diverse functional groups in the side chain. The product 2-alky laziridines which contain various functional group in the side chain can be the precursor of enantiomerically pure functionalized $\beta$-amino alcohols. 1,2-diamines, and nitrogen containing heterocycles.

\section{Experimental}

General. All reactions were carried out under an atmosphere of nitrogen in oven-dried glassware with magnetic stirring. Air sensitive reagents and solutions were transferred wia syringe and were introduced to the apparatus through rubber septa. Tetrahydrofuran (THF) and diethyl ether $\left(\mathrm{Et}_{2} \mathrm{O}\right)$ were distilled from sodium/benzophenone ketyl. Dichloromethane was distilled from calcium hydride. Reactions were monitored by thin layer chromatography (TLC) with $0.25 \mathrm{~mm}$ E. Merck precoated silica gel plates ( $60 \mathrm{~F} 254$ ). Visualization was accomplished with either UV light. or by immersion in solutions of ninhydrine, $p$-anisaldehyde, or phosphomolybdic acid (PMA) followed by heating on a hot plate for about $10 \mathrm{sec}$. Purification of reaction products was carried out by flash chromatography using Kieselgel 60 Art 9385 (230 - 400 mesh). ${ }^{\mathrm{H}} \mathrm{H}-\mathrm{NMR}$ and ${ }^{13} \mathrm{C}-\mathrm{NMR}$ spectra were obtained using a Varian Gemini-300 (300 MHz for ${ }^{1} \mathrm{H}$. and $75 \mathrm{MHz}$ for ${ }^{13} \mathrm{C}$ ). or a Varian Inova -500 $\left(500 \mathrm{MHz}\right.$ for ${ }^{1} \mathrm{H}$. and $125 \mathrm{MHz}$ for ${ }^{13} \mathrm{C}$ ) spectrometer. Chemical shifts are reported relative to chloroform $(\hat{\delta}=7.26)$ for ${ }^{l} \mathrm{H}$ NMR and chloroform $(\hat{o}=77.2)$ for ${ }^{13} \mathrm{C}$ NMR. Data are reported as (br = broad. $\mathrm{s}=$ singlet. $\mathrm{d}=$ doublet. $\mathrm{t}=$ triplet. $\mathrm{q}=$ quartet. $\mathrm{m}=$ multiplet.) Coupling constants are given in $\mathrm{Hz}$. Ambiguous assignments were resolved on the basis of standard one dimen- sional proton decoupling experiments. Optical rotations were obtained using a Rudolph Autopol III digital polarimeter and optical rotation data was reported as follows: $[\alpha]_{0}^{25}$ (concentration $\mathrm{c}=\mathrm{g} / 100 \mathrm{~mL}$, solvent). Elemental analyses were performed by the Organic Chemistry Research Center at Sogang University using a Carlo Erba EA 1180 elemental analyzer. High resolution mass spectra were recorded on a 4.7 Tesla IonSpec ESIFTMS or a Micromass LCT ESI-TOF mass spectrometer. All commercially available compounds were used as received unless stated otherwise

General procedure for the double bond saturation reactions of 2-vinylazinidines. To a solution of 2-vinylaziridine ( 1 equiv) in dry $\mathrm{CH}_{2} \mathrm{Cl}_{2}(0.3 \mathrm{M})$ were added NBSH (4 equiv) and trietlylamine ( 8 equiv) at $0^{\circ} \mathrm{C}$. The reaction mixture was stirred from $0^{\circ} \mathrm{C}$ to RT for $12 \mathrm{~h}$. The mixture became homogenous after 4 $h$ and the reaction nixture was stirred for another $8 \mathrm{~h}$. The reaction was quenched with aqueous sat'd sodium bicarbonate $(10 \mathrm{~nL})$. The organic layer was separated and the aqueous layer was extracted with ethyl acetate $(3 \times 10 \mathrm{~mL})$. The combined organic layers were dried over magnesium sulfate and concentrated in vacuo to yield crude 2-alky laiziridine as an yellow oil. Flash chromatography $\left(\mathrm{SiO}_{2}\right.$. EtOAc/hexanes. $3: 7)$ provided products.

(R)-2-Pentyl-1-[(R)-1-phenylethyl] aziridin (2a): $[\alpha]_{\mathrm{i}}^{\hat{\beta}}+29.5$ (c 0.35. MeOH): ${ }^{l} \mathrm{H}$ NMR (300 MHz. $\left.\mathrm{CDCl}_{3}\right) \delta 7.37-7.25$ (m, $5 \mathrm{H}) .2 .38(\mathrm{q} . J=6.6 \mathrm{~Hz} . \mathrm{lH}) .1 .67(\mathrm{~d}, J=2.7 \mathrm{~Hz} . \mathrm{lH}) .1 .43(\mathrm{~d}$. $J=6.6 \mathrm{~Hz}, 3 \mathrm{H}), 1.4-1.35(\mathrm{~m}, 3 \mathrm{H}), 1.30-1.25(\mathrm{~m}, 3 \mathrm{H}) .1 .11-$ $1.07(\mathrm{nt}, 4 \mathrm{H}), 0.76(\mathrm{t} . J=6.6 \mathrm{~Hz} .3 \mathrm{H}):{ }^{13} \mathrm{C} \mathrm{NMR}(75 \mathrm{MHz}$, $\left.\mathrm{CDCl}_{3}\right)$ oे 144.9. 128.4, 127.5. 127.0. 70.1. 40.9, 33.6, 33.5. 32.0. 27.7, 23.5. 22.9. 14.3: HRMS: $m / z$ calcd for $\mathrm{C}_{15} \mathrm{H}_{25} \mathrm{~N}[\mathrm{M}+$ $\mathrm{Na}{ }^{-} 240.1728$. found 240.1728 .

(R)-2-Octyl-1-[(R)-1-phenylethyll aziridine (2b): $[\alpha]_{\mathrm{I}}^{2}+27.4$ (c 0.8, MeOH): ${ }^{\mathrm{H}} \mathrm{H}$ NMR $\left(500 \mathrm{MHz} . \mathrm{CDCl}_{3}\right) \delta 7.37-7.23(\mathrm{~m}$, $5 \mathrm{H}) .2 .37(\mathrm{q} . J=6.6 \mathrm{~Hz}, \mathrm{lH}) .1 .67(\mathrm{~d} . J=3.0 \mathrm{~Hz}, \mathrm{lH}) .1 .43(\mathrm{~d}$ $J=6.6 \mathrm{~Hz}, 3 \mathrm{H}) .1 .39-1.37(\mathrm{~m}, 2 \mathrm{H}) .1 .30-1.23(\mathrm{~nL}, 4 \mathrm{H}), 1.19-$ $1.10(\mathrm{~m} .5 \mathrm{H}) .1 .1-1.07(\mathrm{~m}, 5 \mathrm{H}), 0.86(\mathrm{t} . J=7.0 \mathrm{~Hz} .3 \mathrm{H}):{ }^{1.5} \mathrm{C}$ NMR (125 MHz. $\left.\mathrm{CDCl}_{3}\right) \delta 145.0,128.4,127.1 .127 .1 .70 .5$. $39.1,34.4,33.1,32.0 .29 .7 .23 .5,29.4,29.3 .27 .6 .27 .4,23.0$. 22.8. 14.3: HRMS: $m / z$ calcd for $\mathrm{C}_{15} \mathrm{H}_{22} \mathrm{~N}$ [M+Na] 282.2198 . found 282.2195 .

(R)-1-[(R)-1-Phenylethyl]-2-tetradecylaziridine (2c): $[\alpha]_{\mathrm{L} i}^{26}$ +44.3 (c 1.0. MeOH); ${ }^{1} \mathrm{H}$ NMR $\left(500 \mathrm{MHz}, \mathrm{CDCl}_{3}\right) \delta 7.38-$ $7.23(\mathrm{~m}, 5 \mathrm{H}) .2 .38(\mathrm{q} . J=6.5 \mathrm{~Hz}, \mathrm{lH}), 1.68(\mathrm{~d}, J=0.3 \mathrm{~Hz}, \mathrm{lH})$. $1.45(\mathrm{~d}, J=6.5 \mathrm{~Hz}, 3 \mathrm{H}), 1.41-1.30(\mathrm{~m}, 2 \mathrm{H}) .1 .31-1.17(\mathrm{~m} .19 \mathrm{H})$. $1.16-1.0(\mathrm{~m} .8 \mathrm{H}), 0.90(\mathrm{t} . J=7.5 \mathrm{~Hz} .3 \mathrm{H}):{ }^{12} \mathrm{C} \mathrm{NMR}(125 \mathrm{MHz}$. $\left.\mathrm{CDCl}_{3}\right)$ oे 145.0, 128.3. 127.1, 127.1. 70.5. 39.1. 34.4. 33.1. $32.1,29.9 .29 .8,29.8,29.8 .29 .8,29.7 .29 .6,29.6 .29 .5,29.3$, 27.4. 22.9. 22.8. 14.3: HRMS: $m / z$ calcd for $\mathrm{C}_{2} \mathrm{H}_{41} \mathrm{~N}[\mathrm{M}+\mathrm{H}]^{-}$ 343.3239. found $34+3319$

(R)-2-Phenethyl-1-[(R)-1-phenylethyl]azinidine $(2 \mathbf{d}):[\alpha]_{\mathrm{II}}^{-6}$ +104.8 (c 0.8, MeOH): ${ }^{1} \mathrm{H}$ NMR $\left(300 \mathrm{MHz}, \mathrm{CDCl}_{3}\right)$ oे $7.40-$ $7.10(\mathrm{~m} .9 \mathrm{H}), 6.89(\mathrm{~d} . J=6.9 \mathrm{~Hz} .1 \mathrm{H}) .2 .50-2.24(\mathrm{~m} .3 \mathrm{H}) .1 .67-$ $1.63(\mathrm{~m} .2 \mathrm{H}) .1 .58-1.55(\mathrm{~m}, \mathrm{IH}) .1 .43(\mathrm{~d} . J=6.6 \mathrm{~Hz} .3 \mathrm{H}) .1 .42-$ 1.39 (n. 2H); ${ }^{12} \mathrm{C} \mathrm{NMR}\left(75 \mathrm{MHz}, \mathrm{CDCl}_{3}\right.$ ) $\delta 145.1,142.1 .128 .5$, $128.3,127.3,127.2,125.7,70.5,38.6,35.1 .34 .2,33.8,23.2$ : HRMS: $m / z$ calcd for $\mathrm{C}_{18} \mathrm{H}_{21} \mathrm{~N}[\mathrm{M}+\mathrm{Na}]^{-} 27+.1572$, found $27+.1570$.

(R)-2-(4-Methylphenethyl)-1-[(R)-1-phenylethyl]azindine 
(2e): $[\alpha]^{31}+1+.4(\mathrm{c} 0.2 . \mathrm{MeOH}) ;{ }^{1} \mathrm{H}$ NMR $\left(300 \mathrm{MHz} . \mathrm{CDCl}_{3}\right) \delta$ 7.38-7.22 (m. 5H). 7.15-7.08 (m 4H). 2.91-2.81 (m. IH). 2.78$2.68(\mathrm{~m} . \mathrm{lH}) .2 .40(\mathrm{q} . J=6.3 \mathrm{~Hz}, \mathrm{lH}) .2 .32(\mathrm{~s} .3 \mathrm{H}) .1 .82-1.71(\mathrm{~m}$. 2H). 1.52-1.44 (m. $5 \mathrm{H}) ;{ }^{13} \mathrm{C} \mathrm{NMR}\left(75 \mathrm{MHz}, \mathrm{CDCl}_{3}\right)$ ò 145.1 . $139.0,135.1,129.0,128.5,128.4,127.3 .127 .2,70.5 .38 .7,35.2$. $34.2,33.3 .23 .2,21.1$; HRMS: $m / z$ calcd for $\mathrm{C}_{1, \mathrm{H}_{23} \mathrm{~N}}[\mathrm{M}+\mathrm{Na}]^{-}$ 288.1728 , found 288.1727

(R)-2-(4-Chlorophenethyl)-1-[(R)-1-phenylethyl]azinidine (2f): $[\alpha]_{D}^{26}+34.5(\mathrm{c} 0.6, \mathrm{MeOH}):{ }^{l} \mathrm{H}$ NMR (300 MHz. CDCl $)$ o $7.39-7.25(\mathrm{~m}, 5 \mathrm{H}) .7 .13-7.11(\mathrm{~m}, 2 \mathrm{H}), 6.77-6.74(\mathrm{~m} .2 \mathrm{H}) .2 .47-$ $2.33(\mathrm{~m} . \mathrm{lH}), 2.30-2.20(\mathrm{~m} . \mathrm{HH}), 1.72-1.62(\mathrm{~m}, 2 \mathrm{H}), 1.55-1.34$ (m. 6H): ${ }^{13} \mathrm{C}$ NMR (75 MHz, $\mathrm{CDCl}_{3}$ ) ò 145.1 . 140.4. 131.4 . 129.9. 128.6. 128.t. 127.t, 127.2, 70.6, 38.3, 34.9. 34.3, 33.1 . 23.2: HRMS: $m / z$ calcd for $\mathrm{C}_{1} \mathrm{H}_{20} \mathrm{ClN}[\mathrm{M}+\mathrm{Na}]^{-} 308.1182$, found 308.1183 .

(R)-2-(4-Nitmphenethyl)-1-[(R)-1-phenylethyl] aziridine (2g): $[\alpha]_{D}^{2]}+46.3(\mathrm{c} 0.4, \mathrm{MeOH}): \mathrm{H}$ NMR $\left(300 \mathrm{MHz}, \mathrm{CDCl}_{3}\right) \delta$ $8.01(\mathrm{~d}, J=8.4 \mathrm{~Hz}, 2 \mathrm{H}), 7.39-7.26(\mathrm{~m}, 5 \mathrm{H}), 6.92(\mathrm{~d} . J=8.4$ $\mathrm{Hz}, 2 \mathrm{H}) .2 .59-2.49(\mathrm{~m} . \mathrm{HH}) .2 .42-2.33(\mathrm{~m} .2 \mathrm{H}) .1 .83-1.7 \mathrm{l}(\mathrm{m}$. 2H). 1.55-1.26 (m, 6H); ${ }^{13} \mathrm{C} \mathrm{NMR}\left(75 \mathrm{MHz}_{2} \mathrm{CDCl}_{3}\right) \delta 149.8$. $144.9,139.9 .129 .2$. 128.6. 127.4, 127.2, 123.5, 70.6. 37.9.34.3. 33.6. 29.8. 23.0: HRMS: $m / z$ calcd for $\mathrm{C}_{18} \mathrm{H}_{31} \mathrm{~N}_{2} \mathrm{O}_{2}[\mathrm{M}+\mathrm{Na}]^{-}$ $319.1+22$, found 319.1423

(R)-2-(2-Iodophenethyl)-1-[(R)-1-phenylethyl]azinidine (2h): $[\alpha]_{\mathrm{D}}^{2 \mathrm{l}}+59.7$ (c 1.65. MeOH): ${ }^{\mathrm{l}} \mathrm{H} \mathrm{NMR}(300 \mathrm{MHz}, \mathrm{CDCl}$ ) o $7.73(\mathrm{~d} J=7.5 \mathrm{~Hz}, \mathrm{lH}) .7 .43-7.23(\mathrm{~m}, 5 \mathrm{H}) .7 .13(\mathrm{t} . J=7.5 \mathrm{~Hz}$. IH). $6.80(\mathrm{t} . J=8 .+\mathrm{Hz}, 2 \mathrm{H}), 2.58-2.48(\mathrm{~m} .2 \mathrm{H}) .2 .45-2.38(\mathrm{~m}$. $2 \mathrm{H}) .1 .78-1.67(\mathrm{~m} .3 \mathrm{H}) .1 .58-1.50(\mathrm{~m} \mathrm{lH}) .1 .44(\mathrm{~d}, J=6.6 \mathrm{~Hz}$, $3 \mathrm{H}):{ }^{13} \mathrm{C} \mathrm{NMR}\left(75 \mathrm{MHz}, \mathrm{CDCl}_{3}\right)$ ò $145.1,1+4.5,139.5,129.5$. $128.5 .128 .3,127.7,127.3,127.1 .100 .5 .70 .5,38.8,38.6 .34 .1$. 33.6. 23.2: HRMS: $m / z$ calcd for $\left.\mathrm{C}_{18} \mathrm{H}_{\Im \mathrm{IN}} \mathrm{IM}+\mathrm{Na}\right]^{-} 400.0538$. found 400.0536 .

(R)-2-(4-Bromo-2-fluorophenethyl)-1-[(R)-1-phenylethyl] azinidine (2i): $[\alpha]_{\mathrm{D}}^{19}+18.5(\mathrm{c} 0.3, \mathrm{MeOH}):{ }^{1} \mathrm{H}$ NMR $(300 \mathrm{MHz}$. $\left.\mathrm{CDCl}_{3}\right) \delta 7.37-7.26(\mathrm{~m} .5 \mathrm{H}), 7.11-7.03$ (m. $\left.2 \mathrm{H}\right), 6.54$ (t. $J=7.8$ $\mathrm{Hz}, \mathrm{IH}) .2 .49-2.22(\mathrm{~m}, 4 \mathrm{H}), 1.70-1.62(\mathrm{~m}, 2 \mathrm{H}), 1.48-1.39(\mathrm{~m}$. $5 \mathrm{H}):{ }^{13} \mathrm{C} \mathrm{NMR}\left(75 \mathrm{MHz}^{2} \mathrm{CDCl}_{3}\right) \delta 162.0 .160 .0,145.1 .131 .9$. $131.9,128.6,127.9,127.4 .127 .2,127.1,127.1,119.6,119.5$. $119.0,118.8,70.5,38.4,34.2,33.3,26.9,23.2$ : HRMS: $m / z$ calcd for $\mathrm{C}_{24} \mathrm{H}_{25} \mathrm{~N}\left[\mathrm{M}+\mathrm{Na}^{-} 370.0583\right.$, found 370.0586 .

3-\{(R)-1-[(R)-1-Phenylethyl]aziridin-2-y $\}$ propanenitrile $(2 \mathrm{j}):[\mathrm{a}]_{\mathrm{I}}^{2 \mathrm{l}}+138.5$ (c 1.0. MeOH): ${ }^{1} \mathrm{H}$ NMR $\left(300 \mathrm{MHz}, \mathrm{CDCl}_{3}\right)$ ò $7.38-7.29(\mathrm{~m}, 5 \mathrm{H}) .2 .44(\mathrm{q}, J=6.3 \mathrm{~Hz}, \mathrm{lH}) .2 .08-1.98(\mathrm{~m}$. lH). 1.84-1.76(m, 3H). 1.53-1.31 (m, 6H); ${ }^{13} \mathrm{C} \mathrm{NMR}(75 \mathrm{MHz}$, $\left.\mathrm{CDCl}_{3}\right) \delta 144.2 .128 .6,127.5 .127 .0,119.4 .70 .1$. 37.0. 34.2. 28.9. 22.2. 15.0: HRMS: $m / z$ calcd for $\mathrm{C}_{13} \mathrm{H}_{16} \mathrm{~N}_{2}[\mathrm{M}+\mathrm{Na}]^{-}$ 223.1211. found 223.1213.

3-\{(R)-1-[(R)-1-Phenylethy]]aziridin-2-y])\} propanenitrile (2k): $[\alpha]_{\mathrm{D}}^{21}+138.5$ (c $\left.1.0, \mathrm{MeOH}\right) ;{ }^{1} \mathrm{H}$ NMR $\left(300 \mathrm{MHz} . \mathrm{CDCl}_{3}\right)$ ô $7.38-7.29(\mathrm{~m} 5 \mathrm{H}) .2 .44(\mathrm{q} . J=6.3 \mathrm{~Hz} \mathrm{lH}) .2 .08-1.98(\mathrm{~m}, \mathrm{lH})$. $1.8+1.76(\mathrm{~m} .3 \mathrm{H}) .1 .53-1.31$ (m. 6H): ${ }^{13} \mathrm{C}$ NMR $(75 \mathrm{MHz}$. $\left.\mathrm{CDCl}_{3}\right) \delta 144.2,128.6 .127 .5,127.0$. 119.4. 70.1. 37.0, 34.2. 28.9. 22.2. 15.0. HRMS: $m / z$ calcd for $\mathrm{C}_{13} \mathrm{H}_{16} \mathrm{~N}_{2}[\mathrm{M}+\mathrm{Na}]+$ 223.1211. found 223.1213.

(S)-2-Pentyl-1-[(R)-1-phenylethyl $]$ azinidine $\left(2 \mathrm{a}^{\prime}\right):[\alpha]_{D}^{21}+64.4$ (c 0.6. MeOH): ${ }^{1} \mathrm{H}$ NMR (300 MHz, $\mathrm{CDCl}_{3}$ ) ò 7.38-7.23 (m. $5 \mathrm{H}), 2.39(\mathrm{q} . J=6.4 \mathrm{~Hz}, \mathrm{HH}), 1.56-1.24(\mathrm{~m} . \mathrm{l}+\mathrm{H}) .0 .92-0.90(\mathrm{~m}$, $3 \mathrm{H}):{ }^{13} \mathrm{C} \mathrm{NMR}\left(75 \mathrm{MHz}, \mathrm{CDCl}_{3}\right) \delta$ 144.9. 128.5, 128.4. 127.0 .
$70.1,40.9,33.6,33.5,31.9,27.7,23.5,22.9,14.3:$ HRMS: $m / z$ calcd for $\mathrm{C}_{15} \mathrm{H}_{23} \mathrm{~N}[\mathrm{M}+\mathrm{Na}]^{-} 240.1728$. found 240.1728

(S)-2-Phenethyl-1-[(R)-1-phenylethyl]azinicine (2b'): $[\alpha]_{\mathrm{j}}^{26}+24.4$ (c $0.25, \mathrm{MeOH}) ;{ }^{1} \mathrm{H}$ NMR $\left(300 \mathrm{MHz} . \mathrm{CDCl}_{3}\right)$ ô 7.37-7.18 (m. $10 \mathrm{H}), 2.95-2.72(\mathrm{~m}, 2 \mathrm{H}) .2 .40(\mathrm{q} . J=6.4 \mathrm{~Hz}, \mathrm{lH}) 1.89-1.69(\mathrm{~m}$. $2 \mathrm{H}), 1.52-1.4+(\mathrm{m}, 5 \mathrm{H}), 1.27(\mathrm{~d}, j=6.0 \mathrm{~Hz} .1 \mathrm{H}) ;{ }^{12} \mathrm{C} \mathrm{NMR}(75$ $\left.\mathrm{MHz}, \mathrm{CDCl}_{3}\right) \delta$ 144.8, 142.1. 128.6, 128.5, 128.5. 127.1, 127.0. $126.0,70.0,40.3,35.0 .34 .2 .33 .6 .23 .5:$ HRMS: $m / z$ calcd for $\mathrm{C}_{18 \mathrm{H}_{21} \mathrm{~N}}[\mathrm{M}+\mathrm{Na}]^{-} 274.1572$. found 274.1574 .

Aclonowledgments. The authors acknowledge the financial support from [KRF-2008-C00481 and NRF-2009-0081956 for W. K. Lee] and Korea Science and Engineering Foundation (R01-2007-000-20037-0, the Center for Bioactive Molecular Hybrides for H.-J. Ha).

\section{References and notes}

1. (a) Tanner, D. Angew. Chem. Int. Ed. Engl. 1994. 33, 599. (b) Pearson, W. H. Lian, B. W.; Bergmeier, S. C. In Comprehensive Heterocuclic Chemistry II; Padwa, A., Ed.; Pergamon Press: New York, 1996: Vol. IA, p. 1. (c) Osbom, H. M. I.: Sweeney, J. B: Tetrahedron. Armmetry 1997, 8, 1693 (d) McCoull, W.: Davis, F. A. Sinthesis 2000, 1347, (e) Zwanenburg. B.: ten Holte, P. In

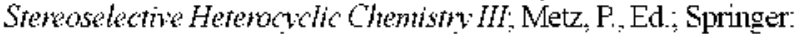
Berlin, 2001, p. 93-124. (f) Sweeney, T. B. Chem. Soc. Rev 2002, 31, 247. (g) Hul, X. E. Tetrahedron 2004, 60,2701.

2. Lee, W. K.: Ha, H.-T Aldrichimica Acta $2003,36,57$

3. (a) Yurn, J. M.; Sim, T. B.; Hahm, H. S.; Lee, W. K.; Ha, H.-T. $J$. Org. Chem 2003, 68, 7675 (b) Jang, S.-Y.; Ha, Y. H.; Ko, S. W. Lee, W.: Lee, I.: Kim, S.: Kim, Y. W.; Lee, W. K.: Ha, H.-J Bioong. Med. Chem. Lett. 2004, 14, 3881 (c) Kim, M. S.; Kim, Y.W.; Hahm, H. S.; Jang, I. W.; Lee, W. K.; Ha, H.-J. Chem. Commun. 2005, 3062. (d) Yoon, H. J.; Kim, Y.-W.; Lee, B. K.; Lee, W. K: Kim Y.; Ha, H.-J. Chent Common 2007, 79. (b) Kim, Y; Ha, H.-T.; Yun, S. Y.: Lee, W. K. Chem. Conmm 2008, 4363

4. Han, S.-M.; Ma, S.-h.: Ha, H.-I.; Lee, W. K. Tetrahedron 2008. 64. 11110 .

5. Lee, B. K.; Kim, M. S.; Hahm, H. S.; Kim, D. S.; Lee, W. K.; Ha, H.-T. Tetrahedron 2006. 62.8393-8397.

6. (a) Hwang, G.-I.; Chung, T.-H.: Lee, W. K. Tetrahedron 1996 , 52, 12111 . (b) Choi, S.-K : Lee, J.-S.; Kim, T.H.; Lee, W. K. $J$. Org. Chem. 1997, 62, 743-745. (c) Bae, T. H.; Shin, S.-H.; Park, C. S.: Lee, W. K. Tetrahedron 1999, 55, 10041. (d) Shin S.-H.: Han, E. Y:; Park, C. S.; Lee, W. K.; Ha, H.-J. Tenahedron: Aswmmeny 2000, 11, 3283. (e) Lee, K.-D.; Sul, J.-M.; Park, T.-H.; Ha, H.-J.; Choi, H. G.; Park, C. S.; Chang, T. W.; Lee, W. K.; Dong, Y; Yur, H. Tetrohtedron 2001, 57, 9655. (f) Park, C. S : Kim, M. S.; Sim, T. B.; Pyun, D. K.: Lee, C. H.; Lee, W. K. J. Oig. Chem. 2003, 68, 43.

7. Unpublished results. Catalytic hydrogenation of 2-vinyl substituted aziridines provides a mixture of ring $\mathrm{C}-\mathrm{N}$ bond reduction product and also side chain reduction product. The $\mathrm{C}(2)-\mathrm{N}$ bond energy difference calculation, between N-methyl-2-ethylaziridine and N-methyl-2-vinylaziridine, was performed on a PC using Spartan 104 Quantum Mechanics Program (PC/X86). The optimization of the geometry of neutral molecules and radicals were preliminarily obtained by a PM3 semiempirical method. The energy minimizations were then perfonmed using BSLYP functional. The $6-31+\mathrm{G}^{*}, 6-31 \mathrm{G}^{*}$ and $6-311+\mathrm{G}^{*}$ were used for all $\mathrm{C}$. $\mathrm{H}, \mathrm{O}, \mathrm{N}, \mathrm{S}, \mathrm{Cl}$ atoms. All geometry optimizations were perfomed without symmetry constraints to ensure that the resultant geometry is not a local minimum. The absolute energies of the calculated species were obtained without corrections for zero point vibrational energy. The homoly tic bond dissociation energy ( $\mathrm{E}^{\mathrm{h} \text { (omal }}(\mathrm{BD})$ ) for all species investigated was calculated using the equation: ( $\mathrm{E}^{\text {homul }}$ $(\mathrm{BD}))=\left[\mathrm{E}^{\mathrm{abs}}\left(\right.\right.$ radical $\left.\left.^{1}\right)=\mathrm{E}^{\mathrm{shs}}\left(\mathrm{radical}^{2}\right)\right]-\mathrm{E}^{\mathrm{ahs}}($ neutral molecule $)$. 
The calculation shows the $\mathrm{C}(2) \mathrm{N}$ bond energy of $\mathrm{N}-\mathrm{m}$ ethyl-2vinylaziridine is $14.9 \mathrm{kcal} / \mathrm{mol}$ less than that of the N-methyl-2ethylaziridine

8. (a) Margathe, J.-F.; Shipman, M.; Smith, S. C. Org. Letr. 2005, 7, 4987-4990. (b) Bussolo, V. D.: Romano, M. R.: Favero, L.; Pineschi, M: Crotti, P. J. Org. Chem 2006, 71, 1696-1699. (c) Mita, T.: Fukuda, N.; Roca, F. X : Kanai, M.; Shibasaki, M. Org. Lett. 2007, 9, 259-262. (d) Hman, J.; Jarevng, T.; Somfai, P. J. Org. Chem. 1996, 61,8148-8159.(e) Ling, R.; Yoshida, M.: Mariano, P. S.J. Org. Chem 1996, 61,4439-4449.

9. (a) Gang, Z: Kainan, Z.: Janis, L. Tetrahedron Lett. 2008, 49 , 6797-6799. (b) Trost, B. M.; Fandrick, D. R. J. Am. Chem. Soc: 2003, 125, 11836-11837. (c) Trost, B. M.; Dong, G. Org. Lett. 2007, 9,2357-2359. (d) Butler, D. C. D.: Inman1, G. A.; Alper, H.
J. Org. Chem. 2000, 65, 5887-5890 (e) Ibuka, T.: Mimura, N.; Aoyama, H.; Akaji, M.; Ohno, H.; Miwa, Y.; Taga, T.; Nakai, K.: Tamamura, H.: Fuji, N.; Yamamoto, Y.J. Org. Chem. 1997, 62, 999-1015. (f) Iska, V. B. R; Gais, H.-T; Tiwari, S. K.; Babu, G. S.; Adrien, A. Tetrohedron Lett. 2007, 48, 7102-7107.

10. Acid labile

11. (a) Michael, H. H.: George. A. O. Oig. Letr. 2002, f, 1771, (b) Cusack, N. T.; Reese, C. B.; Risius, A. C.; Roozpeikar, B. Tetrahedron 1976, 32, 2157. (c) Hunig, S.; Muller, H. R.; Their, W. Angew Chem Intl Ed Engl 1965, 4,271. (d) Myers, A. G; Z Zheng. B.: Movassaghi, M. J. Ong. Chem 1997, 62,7507,

12. (a) Lim, Y.; Lee, W. K. Tetrahedron Lett. 1995, 36, 8431. (b) Chang, J.-W.; Bae, J. H.; Shin, S.-H.; Park, C. S.; Choi, D.; Lee, W. K. Tetrahedron Lett. 1998, $39,9193$. 